\title{
Brain atrophy measurements should be used to guide therapy monitoring in MS
}

Declan Chard

\section{Affiliations}

1. Queen Square Multiple Sclerosis Centre, NMR Research Unit, Department of Neuroinflammation, UCL Institute of Neurology, London, UK

2. National Institute for Health Research (NIHR) University College London Hospitals (UCLH) Biomedical Research Centre, UK

\section{Correspondence to:}

\section{Declan Chard}

Queen Square Multiple Sclerosis Centre, UCL Institute of Neurology, Queen Square, London, WC1N 3BG

Email: d.chard@ucl.ac.uk

Word count: 615

\section{Commentary}

There appears to be little controversy about the use of current brain atrophy measures as a guide to therapeutic decisions in multiple sclerosis (MS): Zivadinov, Dwyer and Bergsland (for), and Barkhof (against), appear to agree that the methods now available are not useable in clinical practice, and that there is much still to be done before they can contribute usefully to treatment choices. I agree with them. However, this does not mean that I think we should give up on brain atrophy measures, given the alternatives, and the emergence of treatments that may actually slow progression in MS. Progressive MS, and the neurodegeneration thought to underly a substantial part of it, is difficult to detect clinically. The onset of progression can take years to become clear [Sand et al. 2014], and in treatment trials much larger cohorts are required to detect effects using clinical compared with 
magnetic resonance imaging (MRI) measures [Miller, Altmann and Chard, 2012]. As such, clinical measures also fall far short of the ideal.

Barkhof, and Zivadinov and colleagues, highlight the mismatch between the established role of brain atrophy measures in early phase treatment trials, and the possible part they may play tailoring treatments to individual people with MS. However, given that the balance between efficacy, side effects and costs differ between treatments, there may be scope to use these measures flexibly in clinical practice. For safe and cost-effective drugs, the thresholds for treatment may be low, seeking to identify the majority who will benefit while still including some who will not. For treatments with a greater risk of harm, or greater costs, the thresholds may be higher, selecting only those who are most likely to benefit.

Another factor to consider is how quickly a decision about treatment needs to be made. In contrast to MS relapses - where events can unfold over hours to days - the consequences of neurodegeneration evolve over months to years. Given this, as Zivadinov et al. observe, while optimally treatments that prevent this would be started as early as possible, brain atrophy measures may still be of value guiding treatment decisions even if they take several years to do so. Relevant to this is the trade off between time and sensitivity to change that is very apparent when we consider the use of brain atrophy measures in clinical trials. Using SIENA - a method that it often used in MS trials - to detect a 50\% treatment effect with $80 \%$ power, over 1 year about 50 people would be required in each treatment arm, while over 2 years this drops to about 30 people [Altmann et al. 2009].

It is also worth recalling how MRI is currently used in MS clinical practice, as a guide to how brain atrophy measures may be used in future. I think it is unlikely that they will be employed in isolation, but rather as an adjunct to, or to augment, clinical and other MRI measures. The McDonald MS diagnostic criteria [Polman et al. 2011] are a superb example of the practical role MRI can play in clinical decision making, and they do not rely solely on MRI findings to establish a diagnosis. Similarly, Rio and colleagues [2009] have shown that a combination of MRI and clinical findings, rather than either alone, is better able to predict disability progression in people with MS taking beta-interferon. However, as noted by Zivadinov and colleagues, and Barkhof, even if we are able to clearly show that brain atrophy measures can contribute usefully to clinical decision making, they still need to be easily implemented on standard MRI machines, readily interpretable by clinicians, and acceptable to regulators. I suspect that we will not see them in use soon. 


\section{References}

1. Sand IK, Krieger S, Farrell C, Miller AE. Diagnostic uncertainty during the transition to secondary progressive multiple sclerosis. Multiple Sclerosis. 2014 Oct;20(12):1654-7.

2. Miller DH, Altmann DR, Chard DT. Advances in imaging to support the development of novel therapies for multiple sclerosis. Clin Pharmacol Ther. 2012 Apr;91(4):621-34.

3. Altmann DR, Jasperse B, Barkhof F, Beckmann K, Filippi M, Kappos LD, et al. Sample sizes for brain atrophy outcomes in trials for secondary progressive multiple sclerosis. Neurology. 2009 Feb 17;72(7):595-601.

4. Polman $\mathrm{CH}$, Reingold SC, Banwell B, Clanet M, Cohen JA, Filippi M, et al. Diagnostic criteria for multiple sclerosis: 2010 revisions to the McDonald criteria. Ann Neurol. 2011 Feb;69(2):292-302.

5. Rio J, Castillo J, Rovira A, Tintore M, Sastre-Garriga J, Horga A, et al. Measures in the first year of therapy predict the response to interferon in MS. Multiple Sclerosis. 2009 Jun 19;15(7):848-53.

\section{Disclosures}

Declan Chard has received honoraria (paid to his employer) from Ismar Healthcare NV, Swiss MS Society, Excemed (previously Serono Symposia International Foundation), Merck, Bayer and Teva for faculty-led education work, and Teva for advisory board work; meeting expenses from Merck, Teva, Novartis, the MS Trust and National MS Society; and has previously held stock in GlaxoSmithKline. 